\title{
A Generalization of the Frobenius Method for Ordinary Differential Equations with Regular Singular Points
}

\author{
Jung-Hua Chou and Raylin $\mathrm{Wu}$ \\ Department of Engineering Science, National Cheng Kung University \\ Tainan, Taiwan 701
}

\begin{abstract}
The conventional Frobenius method for second order differential equations with regular singular points is extended to differential equations of higher and lower orders. The conditions of a point being regular singular are addressed. It is also shown that Cauchy-Euler differential equations are a special case of ordinary differential equations with regular singular points.
\end{abstract}

Key words: Frobenius Method, Ordinary Differential Equations, Regular Singular Points

\section{INTRODUCTION}

The series solution for a regular singular point of an ordinary differential equation with non-constant coefficients is typically addressed for second order linear ordinary equations as:

$$
\frac{d^{2} y}{d x^{2}}+p(x) \frac{d y}{d x}+q(x) y=0
$$

A singular point $x_{0}$ is regular singular if both $\left(x-x_{0}\right) p(x)$ and $\left(x-x_{0}\right)^{2} q(x)$ are analytic at $x_{0}$. Then the series solution of equation (1) about $x_{0}$ can be assumed as:

$y=\sum_{n=0}^{\infty} a_{n}\left(x-x_{0}\right)^{n+r}$

The indicial equation for the exponent $r$ of equation (2) is:

$$
r(r-1)+p_{0} r+q_{0}=0
$$

Where $p_{0}=\lim _{x \rightarrow x_{0}}\left(x-x_{0}\right) p(x)$ and

$$
q_{0}=\lim _{x \rightarrow x_{0}}\left(x-x_{0}\right)^{2} q(x)
$$

The description given above can be seen in books on ordinary differential equations, e.g., [1,2] or advanced engineering mathematics, such as references [3-6]. A natural question is what happens to ordinary differential equations of other orders? Also, what are the conditions for $x_{0}$ to be regular singular for linear ordinary differential equations of orders other than 2? This study explores these issues and provides concrete solutions.

A Simple Starting Point: To begin with, the first order linear ordinary equations of non-constant coefficients are considered. The equations are expressed in the following form:

$\frac{d y}{d x}+p(x) y=0$

A singular point $x_{0}$ of equation (4) can be considered as a regular singular point if $\left(x-x_{0}\right) p(x)$ is analytic at $x_{0}$. Namely, $\left(x-x_{0}\right) p(x)$ can be expanded as:

$\left(x-x_{0}\right) p(x)=\sum_{n=0}^{\infty} p_{n}\left(x-x_{0}\right)^{n}$

And the expansion coefficients are

$$
p_{n}=\left.\frac{1}{n !} \frac{d^{n}\left[\left(x-x_{0}\right) p(x)\right]}{d x^{n}}\right|_{x=x_{0}} ; n=0,1,2, \cdots
$$

Then by assuming $y=\sum_{n=0}^{\infty} a_{n}\left(x-x_{0}\right)^{n+r}$,

equation (4) becomes 


$$
\sum_{n=0}^{\infty}(n+r) a_{n}\left(x-x_{0}\right)^{n+r}+\sum_{m=0}^{\infty} p_{m}\left(x-x_{0}\right)^{m}\left[\sum_{n=0}^{\infty} a_{n}\left(x-x_{0}\right)^{n+r}\right]=0
$$

Or by combing similar terms, it can be further written as:

$$
\sum_{n=0}^{\infty}\left[(n+r)+p_{0}\right] a_{n}\left(x-x_{0}\right)^{n+r}+\sum_{j=1}^{\infty} p_{j} \sum_{n=0}^{\infty} a_{n}\left(x-x_{0}\right)^{n+r+j}=0
$$

From equation (7), it can be seen easily that the coefficient of $x^{r}$ is:

$$
\left[r+p_{0}\right] a_{0}=0
$$

If $a_{0}$ in equation (8) is set to 0 , then the trivial solution $y \equiv 0$ is obtained. Therefore, by not setting $a_{0}$ to 0 , the "indicial equation" in parallel to the second order linear ordinary differential equations denoted by equation (3) is:

$r+p_{0}=0$

As a demonstration purpose, the following simple first order equation is solved by series method.

$$
x \frac{d y}{d x}+y=0
$$

From the above argument, $x_{0}=0$ is a regular singular point and the solution can be assumed as:

$$
y=\sum_{n=0}^{\infty} a_{n} x^{n+r}
$$

Since

$$
p_{0}=\left.\frac{1}{0 !} \frac{d^{0}[x p(x)]}{d x^{0}}\right|_{x=0}=\lim _{x \rightarrow 0} x p(x)=\lim _{x \rightarrow 0} x \frac{1}{x}=1
$$

equation (10) becomes $\sum_{n=0}^{\infty}(n+r+1) a_{n} x^{n+r}=0$

In other words, the indicial equation is $r+1=0$, i.e., $r=-1$ and other coefficients are equal to 0 identically; namely, the solution is:

$y=a_{0} x^{-1}$

As a comparison, the exact solution can be obtained by separation of variables and is:

$y=\frac{c}{x}$

Where $c$ is an integration constant. It can be seen that equations (12) and (13) are essentially the same. It should be pointed out that the Frobenius method can be applied equally well to an ordinary point also as shown by the following example.

$\frac{d y}{d x}+y=0$

Where $x_{0}=0$ is an ordinary point. By assuming $y=\sum_{n=0}^{\infty} a_{n} x^{n+r}$, equation (14) becomes 
$r a_{0} x^{r-1}+\sum_{n=0}^{\infty}\left[(r+n+1) a_{n+1}+a_{n}\right] x^{n+r}=0$.

For a non-trivial solution, the value of $r$ equals to 0 identically and the recurrence relation for the coefficients is

$$
a_{n+1}=-\frac{a_{n}}{n+1}, n=0,1,2, \cdots
$$

And the non-trivial solution is

$$
y=a_{0}\left(1-x+\frac{x^{2}}{2 !}-\frac{x^{3}}{3 !}+\cdots\right)=a_{0} e^{-x}
$$

Which is the exact solution of equation (14). In other words, a series solution of an ordinary point is a special case of the Frobenius solution with $r=0$.

\section{Higher Order Linear Ordinary Differential}

Equations: For higher order linear differential equations,

third order equations are considered first as a starting point of generalization of Frobenius solution as follows:

$$
\frac{d^{3} y}{d x^{3}}+p(x) \frac{d^{2} y}{d x^{2}}+q(x) \frac{d y}{d x}+R(x) y=0
$$

A singular point $x_{0}$ is regular singular if $\left(x-x_{0}\right) p(x) \quad, \quad\left(x-x_{0}\right)^{2} q(x) \quad$ and $\left(x-x_{0}\right)^{3} R(x)$ are analytic at $x_{0}$. Namely, they can be expanded by Taylor series as follows:

$$
\begin{aligned}
& \left(x-x_{0}\right) p(x)=\sum_{n=0}^{\infty} p_{n}\left(x-x_{0}\right)^{n}=\left.\sum_{n=0}^{\infty} \frac{1}{n !} \frac{d^{n}\left[\left(x-x_{0}\right) p(x)\right]}{d x^{n}}\right|_{x=x_{0}}\left(x-x_{0}\right)^{n} \\
& \left(x-x_{0}\right)^{2} q(x)=\sum_{n=0}^{\infty} q_{n}\left(x-x_{0}\right)^{n}=\left.\sum_{n=0}^{\infty} \frac{1}{n !} \frac{d^{n}\left[\left(x-x_{0}\right) q(x)\right]}{d x^{n}}\right|_{x=x_{0}}\left(x-x_{0}\right)^{n} \\
& \left(x-x_{0}\right)^{3} R(x)=\sum_{n=0}^{\infty} R_{n}\left(x-x_{0}\right)^{n}=\left.\sum_{n=0}^{\infty} \frac{1}{n !} \frac{d^{n}\left[\left(x-x_{0}\right) R(x)\right]}{d x^{n}}\right|_{x=x_{0}}\left(x-x_{0}\right)^{n}
\end{aligned}
$$

By assuming a series solution of the form $y=\sum_{n=0}^{\infty} a_{n}\left(x-x_{0}\right)^{n+r}$ and substituting equations (17a)-(17c) into equation (16), the following equation is obtained.

$$
\begin{aligned}
& \sum_{n=0}^{\infty}\left[(n+r)(n+r-1)(n+r-2)+p_{0}(n+r)(n+r-1)+q_{0}(n+r)+R_{0}\right] a_{n}\left(x-x_{0}\right)^{n+r} \\
& +\sum_{j=1}^{\infty} \sum_{n=0}^{\infty} p_{j}(n+r)(n+r-1) a_{n}\left(x-x_{0}\right)^{n+r+j}+\sum_{j=1}^{\infty} \sum_{n=0}^{\infty} q_{j}(n+r) a_{n}\left(x-x_{0}\right)^{n+r+j} \\
& +\sum_{j=1}^{\infty} \sum_{n=0}^{\infty} R_{j} a_{n}\left(x-x_{0}\right)^{n+r+j}=0
\end{aligned}
$$

The indicial equation can be obtained from equation (18) and is 
$r(r-1)(r-2)+p_{0} r(r-1)+q_{0} r+R_{0}=0$

And $p_{0}, q_{0}$ and $R_{0}$ are $\lim _{x \rightarrow x_{0}}\left[\left(x-x_{0}\right) p(x)\right]$,

$\lim _{x \rightarrow x_{0}}\left[\left(x-x_{0}\right)^{2} q(x)\right]$ and $\lim _{x \rightarrow x_{0}}\left[\left(x-x_{0}\right) R(x)\right]$,

respectively.

It is interesting to note that if $x_{0}=0$ is chosen and equation (16) is rewritten into:

$x^{3} \frac{d^{3} y}{d x^{3}}+x p(x) x^{2} \frac{d^{2} y}{d x^{2}}+x^{2} q(x) x \frac{d y}{d x}+x^{3} R(x) y=0$ (20)

And further letting $x p(x)=p_{0}, x^{2} q(x)=q_{0}$ and

$x^{3} R(x)=R_{0}$, then equation (20) becomes a third order Cauchy-Euler equation. It can be readily seen that the indicial equation, equation (19), is the equation obtained by assuming $y=c x^{r}$ as commonly conducted in solving Cauchy-Euler equations. In other words, Cauchy-Euler equation is a special case of linear ordinary differential equations with regular singular points.

The solution methodology can be extended to even higher order linear ordinary equations of order $m$ quite straight forwardly as follows:

$$
\frac{d^{m} y}{d x^{m}}+p(x) \frac{d^{m-1} y}{d x^{m-1}}+q(x) \frac{d^{m-2} y}{d x^{m-2}}+\cdots+z(x) y=0
$$

Then a singular point $x_{0}$ is regular if $\left(x-x_{0}\right) p(x)$, $\left(x-x_{0}\right)^{2} q(x), \ldots$ and $\left(x-x_{0}\right)^{m} z(x)$ are analytic at $x_{0}$. By assuming $y=\sum_{n=0}^{\infty} a_{n}\left(x-x_{0}\right)^{n+r}$ and

converting equation (21) into the following form:

$$
\begin{aligned}
& \left(x-x_{0}\right)^{m} \frac{d^{m} y}{d x^{m}}+\left(x-x_{0}\right) p(x)\left(x-x_{0}\right)^{m-1} \frac{d^{m-1} y}{d x^{m-1}}+ \\
& \left(x-x_{0}\right)^{2} q(x)\left(x-x_{0}\right)^{m-2} \frac{d^{m-2} y}{d x^{m-2}}+\cdots+\left(x-x_{0}\right)^{m} z(x) y=0
\end{aligned}
$$

An equation similar to equation (18) is obtained as given in equation (23).

$$
\begin{aligned}
& \sum_{n=0}^{\infty}\left[(n+r)(n+r-1) \cdots(n+r-m+1)+p_{0}(n+r)(n+r-1) \cdots(n+r-m+2)+\right. \\
& \left.+q_{0}(n+r)(n+r-1) \cdots(n+r-m+3)+\cdots+z_{0}\right] a_{n}\left(x-x_{0}\right)^{n+r} \\
& +\sum_{j=1}^{\infty} \sum_{n=0}^{\infty} p_{j}(n+r)(n+r-1) \cdots(n+r-m+1) a_{n}\left(x-x_{0}\right)^{n+r+j} \\
& +\sum_{j=1}^{\infty} \sum_{n=0}^{\infty} q_{j}(n+r)(n+r-1) \cdots(n+r-m+2) a_{n}\left(x-x_{0}\right)^{n+r+j} \\
& +\cdots+\sum_{j=1}^{\infty} \sum_{n=0}^{\infty} z_{j} a_{n}\left(x-x_{0}\right)^{n+r+j}=0
\end{aligned}
$$


The indicial equation can be deduced from equation (23) as:

$r(r-1) \cdots(r-m+1)+p_{0} r(r-1) \cdots(r-m+2)+\cdots z_{0}=0(24)$

In both equation (23) and (24), $p_{0}, q_{0}, \ldots$ and $z_{0}$ are

$\lim _{x \rightarrow x_{0}}\left(x-x_{0}\right) p(x), \lim _{x \rightarrow x_{0}}\left(x-x_{0}\right)^{2} q(x), \ldots$ and

$\lim _{x \rightarrow x_{0}}\left(x-x_{0}\right)^{m} z(x)$, respectively.

It can be seen that equation (24) gives $m$ roots for $r$ and the indicial equations for lower order linear ordinary differential equations can also be deduced from it. For example, for first order equations, $m=1$, and equation (24) becomes

$$
r+z_{0}=0
$$

A comparison between equation (25) and equation (9) shows that they are essentially the same, except in symbols. By the same token, equations (3) and (19) are special cases for $m=2$ and $m=3$ of equation (24), respectively. Furthermore, it can be observed also that the Cauchy-Euler equation

$x^{m} \frac{d^{m} y}{d x^{m}}+p_{0} x^{m-1} \frac{d^{m-1} y}{d x^{m-1}}+q_{0} x^{m-2} \frac{d^{m-2} y}{d x^{m-2}}+\cdots+z_{0} y=0$

is simply a special case of equation (22).

\section{CONCLUSION}

In this study, a general solution methodology for $m^{\text {th }}$ order linear ordinary differential equations with variable coefficients possessing regular singular points is developed. The Frobenius method and the conditions for a regular singular point of second order differential equations can be deduced by setting $m=2$.

Furthermore, the solution technique for Cauchy-Euler equations can be treated as a special case of this generalized Frobenius method.

\section{REFERENCES}

1. Braun, M., 1975. Differential equations and their applications. An introduction to applied mathematics, Springer-Verlag, Inc.

2. Hollis, S., 2002. Differential equations with boundary value problems. Prentice Hall, Inc.

3. Riley, K. F., M.P. Hobson and S.J. Bence, 1998. Mathematical Methods for Physics and Engineering. A comprehensive guide, Cambridge University Press.

4. Kreyszig, E., 1999. Advanced Engineering Mathematics. $8^{\text {th }}$ edition, John Wiley and Sons, Inc.

5. O'Neil, P.V., 1991. Advanced Engineering Mathematics, $3^{\text {rd }}$ Edn., Wadsworth Publishing Co.

6. Greenberg, M. D., 1998. Advanced Engineering Mathematics. $2^{\text {nd }}$ edition, Prentice-Hall Int., Inc. 